\title{
Analysis of Displays Attributes for use in Avionics Head up Displays
}

\author{
Pooja Verma \\ Department of ECE \\ IGDTUW, Delhi
}

\author{
Vinod Karar \\ Department of ODS \\ CSIR-CSIO, Chandigarh
}

\author{
Vandana Niranjan \\ Department of ECE \\ IGDTUW, Delhi
}

\author{
Surender Singh Saini \\ Department of ODS \\ CSIR-CSIO, Chandigarh
}

\begin{abstract}
Modern avionics provide a comprehensive human-machine interaction. The modern electronic displays are the key components of any glass cockpit based aircraft employing the state of art avionics and are being increasingly used due to two main reasons: firstly, the continuous advancements and improvements in the electronic display technologies, and second being the progressive changes in the onboard data distributing and processing methods in both military and civil aircraft. In this article we have discussed several electronic display devices and relevant technologies for avionics display use especially with reference to the head-up displays. These display technologies have been analysed with reference to the avionics display requirements and vital parameters like size, resolution, brightness, flicker, shades of grey, contrast, color, power consumption, etc.
\end{abstract}

\section{General Terms}

Display Technologies, Avionics Display, Head Up Display

\section{Keywords}

Cathode Ray Tube, Display Attributes, Head Up Display, Display Writing Methods, Display Technologies.

\section{INTRODUCTION}

Avionics is the heart of modern aircraft, and many function of avionics play an important role in both civil as well as military aircraft. A variety of cockpit displays based on various display technologies along with Mission Computers (MC) and Display Processors (DP) provide a great humanmachine interaction. While the MC or DP process the sensors data and provide two types of information to the pilot: One, the data on the demands of aircraft such as pressure, temperature, airspeed, fuel state etc., and second, the data due to the relative situation of aircraft to the outside world such as attitude, navigational position of the aircraft, etc. Keeping these basic functions of the cockpit display functions in mind, variety of cockpit display employed in the modern aircraft such as Multi Functional Displays (MFD), See-through Displays like Head Up Display (HUD) and Helmet Mounted Display (HMD), Moving Map Displays (MMD), Electronic Flight Instrument System (EFIS), etc. have been conceptualized and then custom built as per the application, requirements of the pilot and the aircraft platform [1]-[2].

\section{DISCUSSION ON DISPLAY TECHNOLOGIES SUITABLE FOR AIRCRAFT COCKPIT DISPLAYS}

The light emitting diodes (LEDs) are semiconductor based devices comprising of $\mathrm{p}-\mathrm{n}$ junction diode made from III-V group element. These may include gallium, indium, arsenic, aluminum, phosphorous, or antimony. When the junction is forward bias it emits light due to electron-hole recombination.
These devices are used for backlighting the LCD panels and also used in the LED based navigational, landing and taxi lighting system of aircrafts. However, they are not suitable for direct application as a display device in a cockpit display [1], [3].

The liquid crystal display (LCD) is a non-emissive display technology based on the principle of dynamic scattering of light. Mostly used structure of LCD is twisted nematic. They can result in high contrast ratio coupled with lesser operational voltage requirement and reduced power consumption. However, this technology suffers from the disadvantage such as poor intrinsic viewing angle, backlighting requirements, temperature and sunlight dependent performance, etc. [3]-[5].

The electroluminescent display (ELD) emits light when the electric field is applied across polycrystalline phosphorous surface. This technology can be built to be rugged with large intrinsic viewing angle, higher operational speed, better brightness and contrast. However, it has inherent highly nonlinear voltage-current characteristics, higher operational voltages and low efficiency hence not preferred for avionics use except for aircraft internal lighting purpose but not as a direct display element [3], [6].

The plasma displays are manufactured by sandwiching many cells between two glass plates. By applying electric current to each cell the gas (plasma) is excited which leads to emission of UV rays. This technology is realized in large display sizes which are greater than 32 inches, with high resolution and brightness, excellent contrast and color gamut, large angle of view and higher operational speed. However, its limited operational life and availability in higher sizes only makes it unfit for use in aircraft [3], [7].

The organic light emitting diode (OLED) is a type of LED composed of a film of organic compounds and uses the principle of electroluminance. It overcomes many limitations of LCD like availability in range of sizes including smaller sizes, relatively large viewing angle, high resolution and speed, good color gamut, etc.; however it has got life cycle issues [1] ,[3].

The cathode ray tubes (CRT) were excessively used during the Second World War in the military aircrafts for radar displays to generate alphanumeric characters and symbols. After 1967 many display technologies have been developed with the intention to replace CRT due to its bulky size and higher power consumption, however, for avionic HUD use no technology could fulfill the aircraft requirement like high brightness, contrast ratio and sun-readability, large viewing angle, etc. Hence various types of CRT based on display surface - flat or curved, based on size - range of sizes, based on color - monochrome or color, based on types of phosphor, etc. have been developed over the years [2]. 
The monochrome CRT, which is generally used as display source for avionic HUDs, is composed of one electro gun (cathode) and anode to produce a single color. They are widely used in three types of avionics displays: Head-down display (HDD), HUD and HMD. While HDD uses the raster based CRTs, HUD uses optical thin film beam combiners due to which characters are reflected towards pilot's forward field of view, and optics provides large field of view and narrow wavelength of green so that characters are bright and highly visible. These characters are generated by monochrome CRT having narrow band green color and generated on the principle of stroke writing for high brightness and sharp characters.

Now-a-days, the HUD is used in dual mode so as to provide the information to the pilot in night as well as day time. For day mode it uses stroke writing method while in night mode it uses raster technique for displaying outside world images with stroke writing for symbology generation employed during vertical fly back [8].

The HMD employs raster video during night with high resolution and low brightness, and stroke symbology during day in the same way as used in HUD [2]-[3], [13].

Color CRTs have three electron gun and phosphor screen for red, green, blue color to produce three different brightness values. There are three types of color CRT's available viz. shadow-mask, beam index and Penetron tube. In case of shadow-mask tubes, three phosphors are set in a straight line or alternate fashion in triangular form. In this, invariably masks with dot pitches of $0.2 \mathrm{~mm}$ and beam currents in range of $1.5 \mathrm{~mA}$ are available. Major limitation of this color CRT is the poor efficiency created by the mask as compared to monochrome CRTs because of the availability of three phosphors. The other main issue with shadow-mask tube is in making the mask itself sufficiently rugged as the mask is necessarily thin and fragile causing reduced reliability when subjected to military aircraft's vibration environment.

Alternative of shadow mask tube is the beam index tube to overcome the luminous deficiency of the shadow-mask. This has analogous stripes pattern of phosphor except for the fact that it has electronic method to excite the correct phosphor. Its performance is limited due to many disadvantages like inferior color purity and inferior performance at very low light levels. In addition, it also requires complex drive electronic circuit [2]-[3], [9]. Penetron tube works with only two phosphors, therefore, colors ranging from red through yellow to green can be obtained. Due to the requirement of high voltage switching, they have extra complexity and along with overall poor brightness its application is limited to the cabin environment, for example, air traffic control [2], [10].

The performance requirements of HUD CRT capability of giving line brightness greater than $50,000 \mathrm{Cd} / \mathrm{m}^{2}$ is required to achieve line brightness near $10,000 \mathrm{Cd} / \mathrm{m}^{2}$. The spot size is different in both cursive and raster modes, and optimizing a CRT for both modes rarely feasible. CRT phosphors such as P1, P43 and now p53 are used but whereas the broad spectrum of P1 gave a gentle line broadening the spiked response of p43 and p53 can give rise to color fringing unless optics are fully chromatically corrected [11]- [12].

Now-a-days, an avionics display system has become selfcontained as it provides great human computer interface to get the outside as well as the vital synthetic aircraft and other mandatory parameters necessary of ensuring smooth flight. The integrated cockpit avionics comprises HUD, navigation display, primary flight display, radar display, MFD, mission computers and display processors. They together provide more realistic and real time information [2], [9], [13]

\section{WRITING METHODOLOGY AND DISPLAY ATTRIBUTES FOR AVIONIC DISPLAYS}

A raster CRT graphics device is based on matrix pattern having discrete cells. Each individual cell is made bright by positioning the beam to a given $\mathrm{x}-\mathrm{y}$ coordinate and unblanking the beam. This is also called electromechanical point plotter. These digital coordinates of each cell are converted to analog form to deflect the beam. When the beam is stabilized, it is un-blanked. The raster CRT graphics use a large frame buffer which is connected to a computer memory. The memory is used to store every pixel of the raster. Symbols are generated with the frame buffer one bit at a time. In monochrome CRT, memory bit has only two states namely 0 or 1 while in colour CRTs, frame buffer raster device uses additional bit planes. The CRT image need to be transmitted at the given refresh rate to avoid flicker. The raster driven CRTs are used in MFDs and HDDs [3], [13] - [15].

HUDs and HMDs are run with stroke and raster form of writing methods. In stroke writing, three waveforms are produced namely $\mathrm{x}$ (horizontal deflection), $\mathrm{y}$ (horizontal deflection) waveforms and intensity control $\mathrm{z}$ waveform to form characters and symbols, and the electron beam much like pencil is used to draw symbol. The stroke CRTs needs a short persistency phosphor and high refresh rate to avoid flicker. Apart from CRT, such displays require display buffer to generate symbols on CRT surface and the display controller to refresh continuously the information at the required rate [8].

The factors, which limit the number of vectors drawn on CRT, are governed by the capacity of the display buffer and the refresh rate and speed of the display controller responsible for providing processing speed to the picture information thus deciding on the overall performance. Thus, such kind of display has similar resolution to that of a storage tube display. Various symbol shapes (straight line, arc, etc.) are stored digitally in a library. To generate characters appropriate symbols are called by logical circuitry and fed to a digital to analog converter (DAC). The DAC drives the deflection circuits, which provide the sweep between the coordinate points, and blanking signals are stored with the point coordinate data [15].

There are many display technologies which provide a range of operating characteristics. These characteristics form the basis of selection of particular display technology when compared with respect to the requirements. For head-up display, there are several parameters which need to be considered such as update and request response time, resolution, contrast, brightness, ambient illumination, color properties, sunreadability, night vision imaging system (NVIS) compatibility, flicker rate, and the symbology to be displayed.

The update response time refers to the time between the entry of new data into a display system and the instant the results are ready for display. In systems which display classes of information, this parameter is a function of the priority given new data, the data-input rate and the processing of queues as well as the response time of the display equipment itself. Requirements vary from seconds to hours. The request response time is another vital parameter for avionic displays from an operational and psychological point of view that a display be created soon after the user designates the 
information he/she wishes to be displayed. A request might designate, for instance, the class of information such as airplanes flying over 30,000 feet and the geographic area. The request response time is considered as the time delay from request until the display appears. This is a function of the display access mechanism as well as of the display generation process. These response times are determined in part by display writing speed for individual symbols and complete frames, and the time to remove or erase a display [1], [15].

"The brightness of a viewed object is defined in a psychological sense as a level of light intensity perceived by a viewer. The key physical measure of brightness is luminance. Brightness is defined as the luminance of the brightest component (usually white colour) in the centre of the screen and is measured in candela per square meter $\left(\mathrm{cd} / \mathrm{m}^{2}=\right.$ nit $)$ or foot lamberts $(1 \mathrm{fL}=3.426$ nits)" [1]. In normal room light, the average human eye can discriminate parallel black lines, separated by intervals equal to the line width, when the line separation subtends about 1.5 minutes of arc at the eye. Thus, the eye has an angular resolving power of 40 optical lines (80 TV lines) per degree. From a fixed position, a user can see a plane display which subtends $50^{\circ}$ at the eye. This allows the eye to make out 2000 optical lines of detail on such a display. The eye will be the limiting factor in the amount of usable information that can be presented in a display. In order to perceive further detail, the user must somehow have access to additional displays either on other surfaces or by changing the display (including magnifying portions). Exact resolution requirements vary with display contrast and brightness, information/symbology clutter, background noise (e.g., maps), nature of use (prolonged study or occasional reference), and field of view or the viewing angle [2].

The ratio of the maximum luminance to the minimum luminance that can be generated in the same image, regarded as display contrast ratio, is created by the difference in luminance from two adjacent surfaces and expressed as:

Contrast ratio $\left.=\left(\mathrm{L}-\mathrm{L}_{\mathrm{b}}\right) / \mathrm{L}_{\mathrm{b}}\right)$

Where $\mathrm{L}$ is the display image detail luminance and $\mathrm{L}_{\mathrm{b}}$ is the background luminance.

These factors need to be specified in a predefined illumination environment where ambient light and reflections from the screen will significantly affect the values. A contrast ratio higher than 1.2:1 is required as a minimum for image details detection. An average human eye can perceive millions of different colors. The 1931 Commission Internationale de l'Eclairage (CIE) evolved a three dimensional color space mechanism that allowed any visible color to be mapped. Any color could be located within the color space and its composition from each of the three primary colors viz. red, green and blue can also be manipulated [1].

Readability in a high ambient lighting varying from as low as zero to as high as 1, 00,000 $\mathrm{lx}$ of diffused and/or collimated illumination from one or more directions are the avionic display's typical requirement. The display needs to be mounted so as to minimize the impact of the ambient illumination. Diffusive and specular reflectance must be minimized by optically enhancing the display front surface [1] $-[2]$.

To obtain a possibility to use both the display unit and the Night Vision Goggle (NVG) at the same time, a specific technical solution is selected. Sharing the optical spectra is achieved by using special optical filters on the NVG and the display unit. "NVGs are filtered using a so called minus blue filter, and the display unit is filtered to eliminate excess NIR radiation, so the display can be operated successfully using the naked eye, and without disturbing the NVG" [1].

The critical-fusion frequency, which is the frequency of light flashes, may give an impression to the viewer of a continuous light. The critical fusion frequency is affected by light intensity and bandwidth, length of exposure, brightness of surrounding area. It is also affected by the relative spans of dark and light cycles. Displays formats of data consisting of alphanumeric characters in tabular or straight text form are the most general type of computer output for human interpretation. Requirements often exist for displaying symbols of arbitrary shapes. Symbols provide an extremely efficient means of representing complex thought process, objects or events [15].

These all requirements are fulfilled by CRT based display system for avionics application especially the fighter aircraft electronics where not only the raster kind of display could be produced but also the high intensity alphanumeric characters based on the stroke writing method on various writing speeds.

CRT display writing methods are broadly classified into: (1) Vector or stroke or random scan display, (2) Raster scan display, (3) Interlaced raster scan display, (4) Progressive raster scan display, and (5) Bidirectional raster scan display.

In vector scan, characters or symbols are made by sequences of strokes or short lines. Electron beam is deflected from one end point to the other end point of the lines. It is turned 'on' or 'off' to create patterns as it is deflected across the screen to create the patterns and symbols. Picture information is stored as a set of line drawing commands in memory and these commands are called to control and deflect the beam to 'on' or 'off' in order to draw the lines which will build the required character or symbol. The display is required to be refreshed many times depending on the number of lines to be displayed [16]. In raster scan the electron beam sweeps through entire display screen, one line at a time moving from top to bottom of the screen to display the complete picture information. The intensity values for all the picture elements or pixels on the screen depend on the input to the cathode. The input current intensity controls the electron-beam intensity at different points on screen to display required information. The electron beam moves each line from left to right writing the raster pattern with desired picture information. Subsequently, it returns back to extreme right position by blanking the intensity during retrace. The beam retraces back to the top left of the screen by vertical retrace at the end of the frame [16].

Progressive scan is the way of displaying video information by scanning all lines in a frame in sequence. The scene is scanned a number of times in both directions horizontal and vertical simultaneously to give the illusion of continuous motion. The horizontal deflection coils move the beam across the raster from left to right for the trace. At the end of the line, deflection coils reverses the motion of beam in reverse direction and brings it back to the left by blanking retrace. Vertical deflection coils move the beam to the bottom of screen by vertical trace simultaneously with horizontal deflection. At the bottom position of the CRT screen, vertical deflection coils reverse back the beam to top position of CRT screen through vertical retrace. In contrast to progressive scan, where all lines of a frame are scanned in a sequential manner, a frame is divided into two separate fields in interlaced raster scan. Each field scans alternate lines instead of successive lines, hence, covering half of the total scan lines [8]. 
For a 625 lines PAL system, the frame is divided into two fields of 312.5 lines each. Odd field ends at the centre of bottom line and the even field starts from the center of top line. Interlaced Scanning reduces the flicker to acceptable level as display is scanned twice the same rate as in progressive scanning [8].

Bidirectional raster scanning employs the use of horizontal retrace as trace. There is no horizontal retrace in bidirectional scan. Whenever the electron beam reaches the right edge of the CRT screen, it reverses back to the left edge of CRT screen again tracing the CRT screen with desired picture information. Both, the two conventional raster drawing methods progressive and interlaced scan methods scan display screen in only one direction, they have different scanning mechanisms [17].

Table 1. Display technologies and their features comparison

\begin{tabular}{|c|c|c|c|c|c|c|c|}
\hline Features & $\begin{array}{c}\text { AML } \\
\text { CD }\end{array}$ & CRT & ELD & PDP & OLED & LED & FED \\
\hline Resolution & H & H & M/H & M/H & H & H & M/H \\
\hline Luminance & H & H & P & P & P & H & H \\
\hline $\begin{array}{c}\text { Contrast } \\
\text { Ratio }\end{array}$ & H & H & H & H & H & H & H \\
\hline $\begin{array}{c}\text { Ambient } \\
\text { Contrast }\end{array}$ & H & H & M & M & M & H & H \\
\hline Life Time & H & H & H & H & P & H & H \\
\hline $\begin{array}{c}\text { Temperature } \\
\text { Range }\end{array}$ & N & W & W & M & N & W & W \\
\hline $\begin{array}{c}\text { Viewing } \\
\text { Angle }\end{array}$ & M & H & H & H & M & H & H \\
\hline Active Area & H & H & M & M & M & M & M \\
\hline $\begin{array}{c}\text { NVIS } \\
\text { Compatibility }\end{array}$ & H & H & M & H & M & H & H \\
\hline $\begin{array}{c}\text { Colour } \\
\text { Gamut }\end{array}$ & $\mathrm{H}$ & $\mathrm{H}$ & $\mathrm{P}$ & $\mathrm{H}$ & $\mathrm{H}$ & $\mathrm{P}$ & $\mathrm{H}$ \\
\hline $\begin{array}{c}\text { Screen } \\
\text { Update Time }\end{array}$ & $\mathrm{H}$ & $\mathrm{H}$ & $\mathrm{M}$ & $\mathrm{H}$ & $\mathrm{H}$ & $\mathrm{H}$ & $\mathrm{H}$ \\
\hline
\end{tabular}

\section{H - High, L - Low, M - Medium, P - Poor, W - Wide, N - Narrow}

The monochrome $\mathrm{P} 1, \mathrm{P} 43$ or $\mathrm{P} 53$ phosphor HUD CRT supports the stroke (vector) as well as various formats of raster. As evident from Table 1, the CRT scores well in all aspects of HUD display requirements especially for aircraft and there also especially for fighter aircrafts. The paramount parameters of brightness, contrast ratio, line width and support for various writing methods are met by CTT technology as discussed in the above sections.

\section{CONCLUSION}

Various parameters related to avionics display especially HUDs for fighter aircrafts were discussed. It emerged that CRT has several advantages in terms of excellent resolution, excellent brightness and contrast ratio required during the day flights where the background could be sun-lit diffused clouds, reasonable life time, excellent temperature stability, no issues with the viewing angle, easier to achieve NVIS compatibility and very good color gamut. This in addition to its capability of supporting stroke as well as various methods of raster writing makes CRT still a perfect choice for fighter aircraft HUDs.

\section{ACKNOWLEDGMENTS}

We thank Director, CSIR-CSIO Chandigarh, India for supporting the research reported in this paper.

\section{REFERENCES}

[1] Livada, B., "Avionic Displays", Scientific Technical Review, 62(3-4), 70-79, 2012.

[2] Hunt G. H., "Airborne electronic displays", Communications, Radar and Signal Processing, IEE Proceedings F 128.7: 465-483, 1981.

[3] Chahal Nidhi, Vinod Karar and Manpreet Singh. "Study of Writing Methodologies for Graphical Display on Flat Cathode Ray Tube", International Journal of Computer Applications, 74.7: 29-32, 2013.

[4] Clark M. G., "Liquid crystal displays and color switches”, Displays, 1, pp. 17-24, 1979.

[5] http://www.dolcera.com/wiki/index.php?title=LCD_Tec hnologies.

[6] Kazan B., "Electroluminescent displays", Proc. S.I.D., 17, pp. 23-29, 1976.

[7] Willson R. H., “The plasma display”, Inf. Disp.,5, pp. 39,

[8] Gulati, R. R. Monochrome and colour television. New Age International, 2007.

[9] Atkin K. N., "Recent Advances in Cockpit Aids for Military Operations", Defence Science Journal, 38.3 (2014): 231-243, 2014

[10] Woodcock S., and Leyland J. D., "The choice of phosphor for modern CRT display applications", Displays, 1, pp. 69 - 82, 1979.

[11] Larach S., and Hardy A., "Cathode-ray-tube phosphors: principles and applications", Proc. IEEE, 61, pp. 915926, 1973.

[12] http://rochesteravionicarchives.co.uk/img/catalog/ZZ_13 68362369_DDBR0080\%20(O\&A-1b).pdf

[13] Brandtberg Hans. "Electronic display system for the Swedish JAS 39 Gripen aircraft", Aerospace and Electronics Conference, 1994. NAECON 1994, Proceedings of the IEEE 1994 National. IEEE, 1994.

[14] A. Bowman and J. B. Gosling, "Symbol generator for a graphical-display system", England PROC. IEEE, Vol. 122, No. 11, November 1975.

[15] Loewe, R. T., R. L. Sisson, and P. Horowitz. "Computer generated displays."Proceedings of the IRE 49.1: 185$195,1961$.

[16] Snehi, Jyoti. Computer peripherals and interfacing. Firewall Media, 2006.

[17] Spitzer, Cary R. Avionics: Elements, software and functions. CRC Press, 2006.

[18] Chorley R. A., "Seventy years of flight instruments and displays", Aeronautical J., 80, pp. 323-342 1976.

[19] Streetly M., "Confound and destroy", Macdonald and Jane's, London, 1978.

[20] Oldfield D. E., "Initial trials in Hunter XE531 of an electronic display of basic flight data", Royal Aircraft Establishment Technical Memorandum IEE 177, 1967. 
[21] Special Issue on the application of colour to displays, Proc. S.I.D., 20, 1979.

[22] De Haan E. F. and Weimer K. R. V., "The beam indexing colour television-display tube", Roy. Telev. Soc. J., 11, pp. 278-282, 1967. 1968.

[23] Hasker J. and De Dlerk J. J. M., "Improved electron gun for the beam-indexing colour-television display", IEEE Trans., ED-20, pp. 1049-1052, 1973.

[24] Seats P., "The cathode-ray tube - a review of current technology and future trends", IEEE Trans., ED-18, pp. 679-684, 1971.

[25] http://en.wikipedia.org/wiki/Cathode_ray_tube 